\title{
Sensing the optical properties of diffusive media by acousto-optic pressure contrast imaging
}

\author{
Puxiang Lai, Ronald A. Roy and Todd W. Murray \\ Department of Mechanical Engineering, Boston University \\ 110 Cummington Street, Boston, MA 02215 USA
}

\begin{abstract}
Acousto-optic imaging (AOI) is a dual-wave modality that combines ultrasound with diffuse light to achieve deep-tissue imaging of optical properties with the spatial resolution of ultrasound. Progress has been made in the detection of optically absorbing inhomogeneities in recent years, yet it remains a challenge for AOI to detect targets possessing low scattering contrast and to obtain quantitative measurement of optical properties at depth with high resolution. A new photorefractive crystal (PRC) based AOI system operating in the near-infrared optical wavelength was developed and optimized. Based on relative changes in the AOI response induced by different acoustic pressures, we now propose a new sensing and imaging modality, pressure contrast imaging (PCI), to enhance and quantify the detection of scattering inhomogeneities. It is demonstrated experimentally that the image contrast information obtained with the new approach is independent of the background light intensity and the details of the optical collection components and potentially allows for an accurate and quantitative characterization of the media's spatially dependent optical properties.
\end{abstract}

Keywords: acousto-optic imaging, ultrasound-modulated optical tomography, photorefractive crystal, scattering contrast, pressure ratio, tissue characterization

\section{INTRODUCTION}

The optical properties of biological tissue, such as the absorption and reduced scattering coefficients, are influenced by the functional state of tissue and tissue microstructure, and local variations in optical properties can be used to detect and diagnose the presence of tissue abnormalities or diseased states [1]. Optical approaches to sensing and imaging in biological media are, in addition, attractive in that they are non-invasive and non-ionizing. Optical imaging in biological tissue is, however, complicated by high optical scattering that results in a reduction of spatial resolution as the imaging depth is increased. In an effort to circumvent this difficulty, a hybrid imaging modality called acousto-optic imaging (AOI) was proposed and developed [2-4]. In AOI, the sample is illuminated with a continuous wave (CW) laser source and simultaneously subjected to a pulsed or continuous wave ultrasound field. As the light propagates through the sample media, it interacts with the ultrasound through both the ultrasound induced displacement of optical scatterers and the

Further author information: Puxiang Lai, lpx@bu.edu, 617-353-3955

Prof. Ronald A. Roy, ronroy@bu.edu, 617-353-4846

Prof. Todd W. Murray, twmurray@bu.edu, 617-353-3951 
pressure induced changes in refractive index [5,6]. These effects combine to result in a net phase or frequency shift of the light that has traversed the ultrasound field. The magnitude of the phase shift imparted to the light gives an indication of the local optical properties in the acousto-optic (AO) interaction region, defined as the region over which the light and sound interact. The net result is the detection of optical property contrast at depth in biological media with a spatial resolution approaching that of ultrasound imaging.

One particularly difficult challenge in AOI is obtaining sufficient sensitivity to detect small optical contrast deep within biological tissue. Only the light in the interaction region is affected by the ultrasound, and this is typically detected against a much stronger background of unmodulated light. In addition, the phase shift is spatially incoherent in that it depends on the particular path that the light travels through the ultrasound field. Finally, the total light that can be collected after propagation through diffuse media is small. In early setups [4, 7], one or a few speckles were collected and sent to a photodetector where the intensity modulation was detected. The low light levels inherent in such an approach limit the sensitivity. More recently, several techniques for the improved detection AOI signals have been proposed and demonstrated. Full field detection with a CCD array [8, 9] improves the signal-to-noise ratio (SNR) over using a single photodetector by a factor of $\sim \mathrm{N}^{1 / 2}$ ( $\mathrm{N}$ is the number of array pixels). Detection schemes incorporating photorefractive crystal (PRC) based interferometers [10-13] and confocal Fabry-Perot interferometers (CFPI) [14] have also been developed. Finally, a technique based on spectral-hole burning has been presented recently [15]. The interferometry and SHB approaches are capable of delivering a fast response time and large optical etendue.

In AOI, the phase (or frequency) shift imparted on the light by the ultrasound is converted by an intensity modulation through a given optical detection scheme, and the strength of this intensity modulation, or AO signal, is used to infer information about the optical properties of the AO interaction region. The strength of the AO signal is furthermore dictated by the intensity of light in the AO interaction region and the characteristics of the ultrasound field. In addition, this signal is also affected by, among other things, the propagation path of light to the AO interaction region, the propagation from the $\mathrm{AO}$ interaction region to the optical detection system, the total amount of light collected, and the characteristics of the demodulation scheme. While direct AOI imaging is certainly useful for the detection of optical contrast, it is less suitable for the quantitative measure of the optical properties. One technique for obtaining quantitative information from the AOI response is to measure the responses at multiple optical wavelengths [16] and use the spectral information to infer, for example, the composition of the media in the AO interaction region. While quantitative optical property information is possible in this case, it still requires some approach to normalize out the difference in, for example, photon mean free path outside of the inclusion at the different wavelengths.

In this paper, a new approach to imaging using the $\mathrm{AO}$ response, referred to as pressure contrast imaging (PCI), is demonstrated. A GaAs photorefractive crystal based interferometer is used to measure the AO signal generated by insonifying tissue phantoms with short pulses of ultrasound at two different ultrasound pressures. The two signals are then divided to yield a pressure contrast response that is solely dependent on the mean phase shift imparted on the light by the ultrasound, the ultrasound pulse shape, and the pressures $\left(\mathrm{P}_{1}\right.$ and $\left.\mathrm{P}_{2}\right)$ used. For a given ultrasound pulse shape and pressure amplitudes, the pressure contrast ratio can therefore be directly related to the optical properties of the target material. In this paper, we focus on the measurement of the reduced scattering coefficient of homogeneous tissue phantoms. We also demonstrate the quantitative measurement of the reduced scattering coefficients of inclusions 
exhibiting scattering contrast with the surrounding media.

\section{EXPERIMENTAL SETUP AND PRESSURE CONTRAST IMAGING}

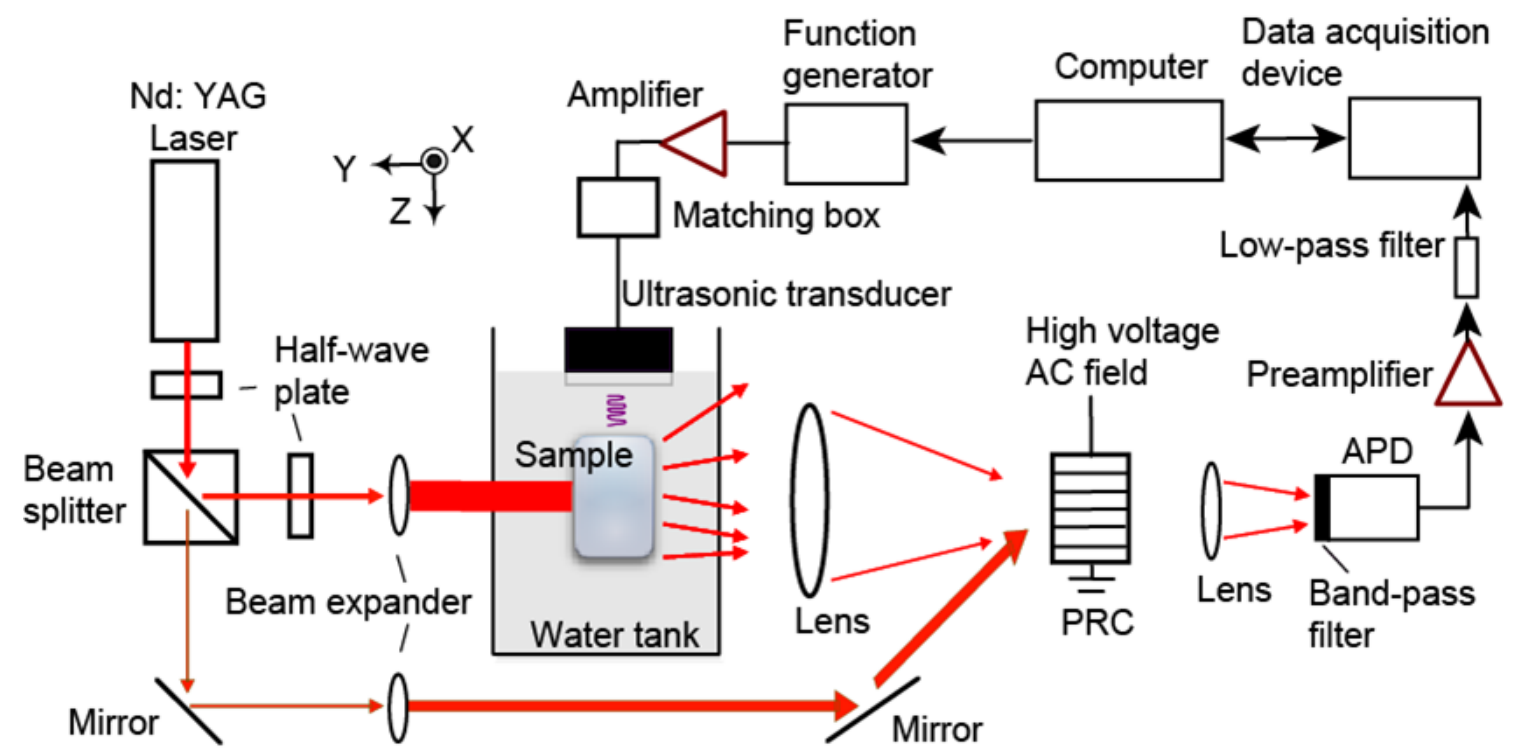

Fig. 1. Experimental setup used for pressure contrast imaging.

The experimental setup is illustrated in Fig. 1. The output of a 1064-nm wavelength Nd: YAG laser (CrystaLaser, CA) is split into a signal beam and a reference beam. The signal beam is expanded and propagates along the negative- $Y$ axis through the walls of the test tank to the polyacrylamide tissue phantom. The phantom is submerged in degassed water and the intensity of light incident on the phantom surface is approximately $200 \mathrm{~mW} / \mathrm{cm}^{2}$ (ASNI limit for safe clinical usage is $1 \mathrm{~W} / \mathrm{cm}^{2}$ at this wavelength [17]). Light gets diffused in the phantom and phase modulated by the ultrasound within the AO interaction region. The scattered signal beam is collected and directed into the PRC where it interferes with the reference beam. The intensity of the reference beam at the PRC is approximately $15 \mathrm{~mW} / \mathrm{cm}^{2}$. A GaAs photorefractive crystal (MolTech GmbH, Berlin, Germany) with dimension $7 \times 7 \times 7 \mathrm{~mm}^{3}$ is used, and a 2.1-KHz, 5-KV peak-to-peak AC electric field is applied to the crystal to enhance the two-wave-mixing (TWM) gain. The response time of the crystal is typically $5-10 \mathrm{~ms}$ under our experimental conditions. The light exiting the crystal is sent through a $1064 \mathrm{~nm}$ bandpass filter to a 5-mm diameter avalanche photodetector (APD, Pacific Silicon Sensor, CA). The signal from the APD is pre-amplified (10x), low-pass filtered $(<500 \mathrm{kHz})$, digitized, and sent to a personal computer for additional processing. The ultrasound source is a spherical single element focused transducer (Sonic Concepts, H101) with a cigar-shaped focal region $1.5 \times 1.5 \times 9 \mathrm{~mm}^{3}$ along XYZ-axis. A function generator (Agilent, 33120A) excites pulse trains, which are amplified with a power amplifier (ENI, A150) and then sent to an impedance matching box before driving the transducer. Unless otherwise mentioned, $1.0 \mathrm{MHz}, 3$-cycle acoustic bursts propagating along the Z-axis with a pulse repetition frequency (PRF) of 1-KHz are employed to generate the AOI response in this study.

The general operation of the detection system has been described in literature $[10,18,19]$, and here we briefly review the nature of detected signals. In our setup, the transmitted signal beam and diffracted reference beam exiting the PRC are 
phase-front matched and interfere constructively at the detector. In the presence of ultrasound induced phase modulations, a decrease in the light measured at the photodiode is expected, regardless of the sign of phase modulation. The AO signal observed using photorefractive detection follows the general form:

$$
I=2 I_{S O} \exp (-\alpha L) \exp (\gamma L) f(t) \frac{1}{N} \sum_{j=1}^{N}\left[1-J_{0}\left(\left|\Phi_{j}\right|\right)\right]
$$

where $I_{s o}$ is the signal beam intensity incident on crystal surface, $\alpha$ and $L$ are the absorption coefficient and length of the crystal, respectively, $\gamma$ is the TWM gain coefficient, $f(t)$ is the normalized envelope of the phase modulation (corresponding to the envelope of ultrasound burst), $\Phi_{j}(P)$ is the accumulated phase shift along a given optical path at an ultrasound pressure $\mathrm{P}$, and $\mathrm{N}$ is the total number of optical paths. In stating the equation in this form, we assume that the light incident on the AO interaction region is uniform over space. The presence of ultrasound is seen to produce a net shift, or DC offset, in the measured intensity. Referring back to equation 1, we can see that only the last term, the accumulated phase shift, is affected by the ultrasound pressure. Taking the ratio of AOI signals taken at two different pressures yields:

$$
S_{P R}=\frac{I_{P_{1}}}{I_{P_{2}}}=\frac{\sum_{j=1}^{N}\left[1-J_{0}\left(\left|\Phi\left(P_{1}\right)_{j}\right|\right)\right]}{\sum_{j=1}^{N}\left[1-J_{0}\left(\left|\Phi\left(P_{2}\right)_{j}\right|\right)\right]} .
$$

Equation 2 shows that the pressure contrast response is independent of the total light collected at the PRC, as well as the characteristics of the detection system. The pressure ratio is only dependent on the shape of the ultrasound pulse and the optical properties of the acousto-optic interaction region. In this paper, we show that the AOI system may be calibrated at $\mathrm{P}_{1}$ and $\mathrm{P}_{2}$ for a particular ultrasound pulse shape, allowing for a quantitative measure of the reduced scattering coefficient. Once calibrated, the system can then be used to measure the reduced scattering coefficient of a buried inclusion as well as a homogeneous medium. The ultrasound pulse shape varies spatially due to the fact that a focused transducer is used. For quantitative measurements, the AO signal at the ultrasound focus is used. The focal point must be scanned through the region of interest to create an image.

\section{RESULTS AND DISCUSSION}

The AOI signal strength measured as a function of ultrasound focal pressure across a homogeneous phantom with a reduced scattering coefficient of $7 \mathrm{~cm}^{-1}$ and dimensions $40 \times 25 \times 40 \mathrm{~mm}^{3}$ along XYZ-axes is shown in Fig. 2. For this experiment, a 3-cycle ultrasound pulse was used to elicit the AOI response. At each pressure, AOI waveforms were averaged over 20,000 sweeps and this process was repeated 8 times to obtain a mean AOI signal, from which the AOI strength was recorded. The data is fit with the function:

$$
I=\eta\left[1-J_{0}\left(\beta P_{f}\right)\right]
$$


where $\eta$ and $\beta$ are constants and $\mathrm{P}$ is the focal pressure. Here $\eta=2 I_{S O} \exp (-\alpha L) \exp (\gamma L) f(t)$ (see Eq. 1) and $\beta \mathrm{P}_{\mathrm{f}}$ is a measure of the mean phase shift under the assumption that this phase shift is linearly proportional to pressure. The coefficient $\beta$ is directly related to the optical properties of the AO interaction region. Equation 3 shows excellent agreement with the experimental data, although we note that it is only equivalent to Eq. 1 under the condition: $\frac{1}{N} \sum_{j=1}^{N}\left[1-J_{0}\left(\left|\Phi_{j}\right|\right)\right] \approx\left(1-J_{0}\left(\beta P_{f}\right)\right)$, meaning that the accumulated phase shift along each path is equal $\Phi_{j}=\Phi=\beta P_{f}$. Nevertheless, Eq. 3 provides a useful means for interpreting the experimental measurements in that measurement of the pressure ratio can be used to extract a value for the constant $\beta$, with a large value of $\beta$ indicating large phase shifts induced by the ultrasound.

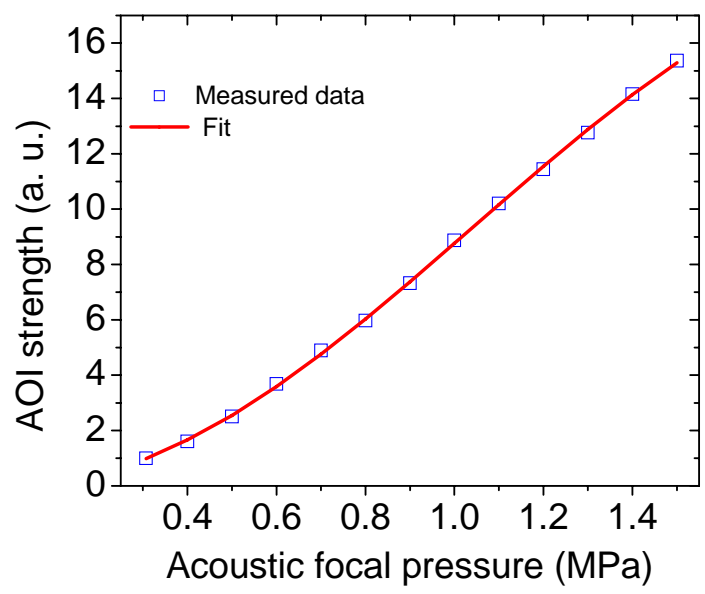

Fig. 2. Measured AOI strength as a function of applied focal pressure in a homogeneous phantom $\left(\mu_{s}^{\prime}=7 \mathrm{~cm}^{-1}\right)$. The discrete squares are measured AOI strength and the line is a curve fit using the function $I=\eta\left[1-J_{0}\left(\beta P_{f}\right)\right]$.

In order to evaluate the pressure contrast response as a function of reduced scattering coefficient, nine homogeneous polyacrylamide gel phantoms, all sized $40 \times 25 \times 40 \mathrm{~mm}^{3}$ along XYZ-axes, were fabricated with a same optical absorption (around $0.1 \mathrm{~cm}^{-1}$ ) but different reduced scattering coefficients (from 2 to $10 \mathrm{~cm}^{-1}$ ) by changing the concentration of $0.7-\mu \mathrm{m}$-diameter polystyrene microspheres. The appropriate particle concentration was determined using a web-based Mie scattering calculator [20]. Three-cycle ultrasound bursts at focal pressures of $1.5 \mathrm{MPa}$ and $0.3 \mathrm{MPa}$ were used to generate the AOI responses at $\mathrm{P}_{1}$ and $\mathrm{P}_{2}$, respectively. The ultrasound transducer was positioned such that the focal region was roughly at the center of the phantoms. Two AOI signals, recorded at the point when the ultrasound pulse reached the transducer focus, were acquired by coherently averaging over 10,000 sweeps, and the pressure ratio determined according to Eq. 1. The measurements were repeated 80 times to obtain a mean $S_{P R}$ with a low standard error. The same procedure was conducted for all phantoms.

Fig. 3(a) shows the measured pressure ratio as a function of medium diffusivity. The pressure ratio is seen to increase with the reduced scattering coefficient. Figure 3(b) shows the value of $\beta$ obtained through approximating the AOI response according to Eq. 3. The reduction of $\beta$ with increased scattering coefficient can be interpreted as a decrease in 
the mean phase shift imparted on the light by the ultrasound as the reduced scattering coefficient is increased. This finding is in qualitative agreement with measurements on scattering samples reported in the literature [21, 22]. It is important to note that a) the pressure ratio is independent of the amount of light collected by the detection system and b) the pressure ratio is independent of the position of the ultrasound focus within the homogeneous phantom so long as any spatial variations in the light distribution within the phantom are large with respect to the ultrasound pulse (i.e., the ultrasound pulse should be uniformly illuminated). The former finding was confirmed by moving the ultrasound focus along both the optical and ultrasound axes and measuring the pressure ratio at the focus. In both cases, the results were consistent with Fig. 3(a). The pressure ratio approach can thus be used as a relatively simple means of measuring the reduced scattering coefficient of homogeneous tissue phantoms or biological media.

(a)

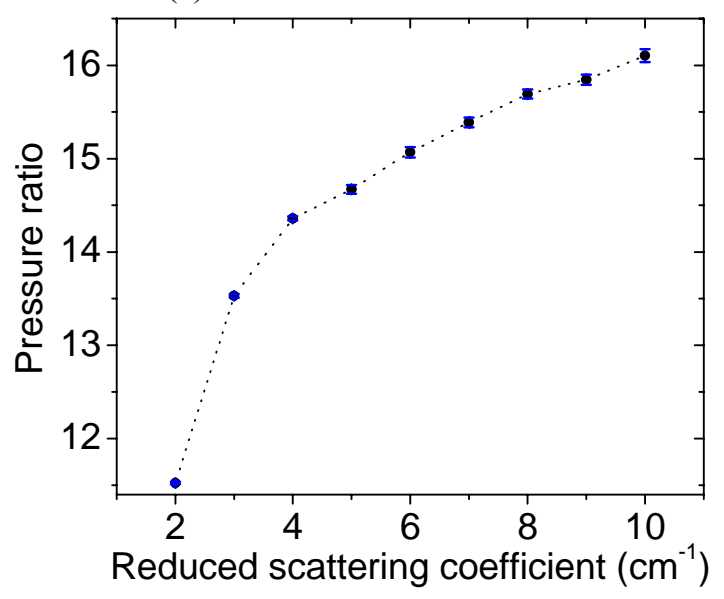

(b)

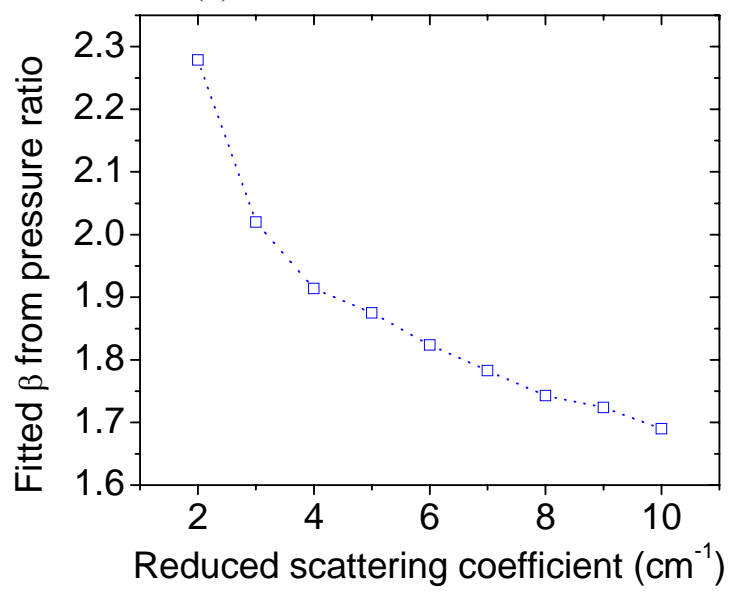

Fig. 3. (a) Pressure ratio and (b) the corresponding $\beta$ as a function reduced scattering coefficient.

The pressure contrast approach was also used to detect and characterize a scattering inclusion embedded in a tissue phantom. The experimental configuration is shown in Fig. 4(a). A phantom sized 40x25x40 $\mathrm{mm}^{3}$ (along the XYZ-axes) with a reduced scattering coefficient about $7 \mathrm{~cm}^{-1}$ was prepared in the same way as the previous homogeneous phantoms, except that at the center of the phantom a $5 \times 6 \times 20 \mathrm{~mm}^{3}$ (along XYZ-axes) inclusion with a reduced scattering coefficient of $10 \mathrm{~cm}^{-1}$ was embedded. The inclusion was made of the same acrylamide gel as the background material. The ultrasound pulses were fired from the top and travelled downwards ( $Z$ axis in Fig. 4(a)), and the center of acoustic focal region was placed in the middle of the phantom (and the inclusion) along the Z- direction. During the experiment, the phantom was scanned along the $X$ direction (which is out of the plane in Fig. 1) with a step size of $1 \mathrm{~mm}$, while the optical and acoustic sources and the detection components remained stationary. At each position the pressure ratio was obtained using the same procedure outlined for the measurements reported in Fig. 3, except that the measurement was repeated 8 times at each point. The inclusion is of sufficient size along the Z-direction that when the ultrasound pulse traverses the inclusion, the entire ultrasound packet will be inside of the inclusion. In other words, the detected pressure ratio at the focus when the ultrasound traverses the inclusion should be consistent with that observed in a phantom with a reduced scattering coefficient of $10 \mathrm{~cm}^{-1}$, and not affected by the background medium. 

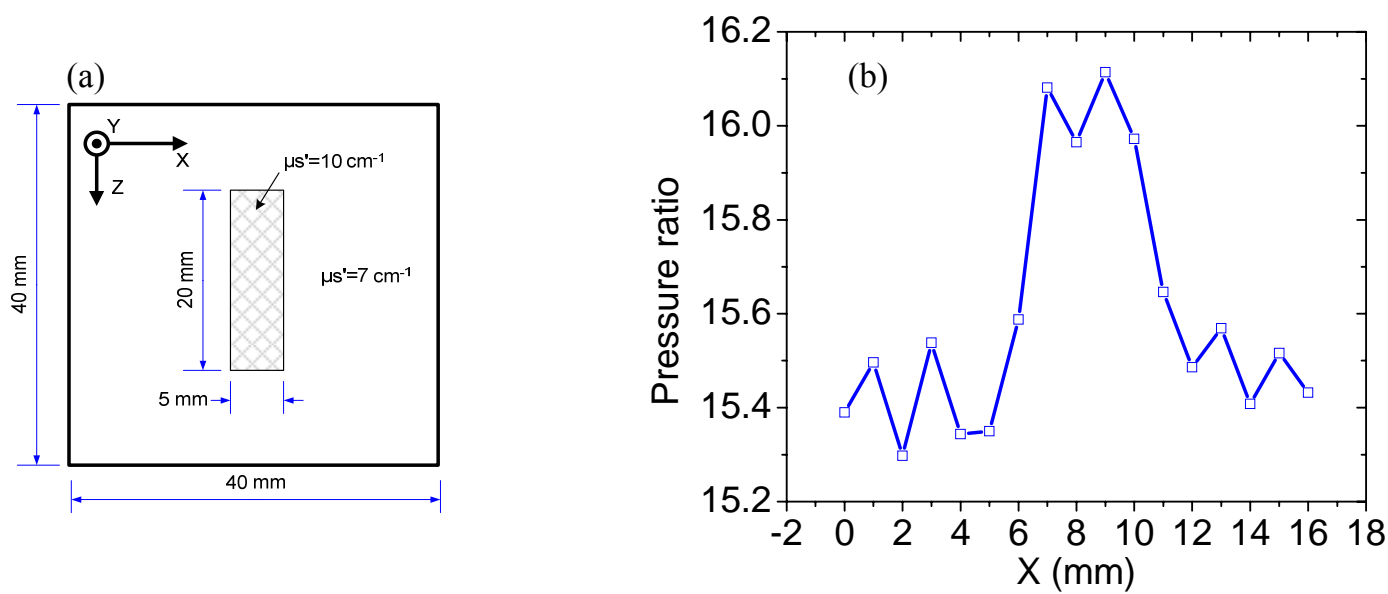

Fig. 4. (a) A highly diffuse phantom (25 mm along Y-axis) with one an embedded inclusion (6 mm along Y-axis) at the center; (b) The pressure ratio as a function of phantom position along the $\mathrm{X}$ direction.

Figure 4(b) shows the resulting pressure ratio $P_{r}$ as a function of phantom position in the $\mathrm{X}$ direction. A scattering region with higher diffusivity than the surrounding material is observed between $6 \mathrm{~mm}$ and $12 \mathrm{~mm}$, which is reasonable considering the size of the inclusion and the lateral width of ultrasound pulses. Outside of the inclusion, pressure ratio values in the 15.30-15.55 range are observed, while in the inclusion we find pressure ratio values in the 15.95-16.15 range. Referring back to the data in Fig. 3(a), these values are consistent with materials with media with reduced scattering coefficients of 7 8 and $10 \mathrm{~cm}^{-1}$, respectively. This result further confirms that the pressure ratio is not dependent on the characteristics of the optical path that the light follows or the amount of light collected, but rather is dictated by the optical properties within the region where the light and sound interact. An inclusion with a reduced scattering coefficient of $10 \mathrm{~cm}^{-1}$ gives a similar pressure contrast response as a homogeneous phantom of reduced scattering coefficient of $10 \mathrm{~cm}^{-1}$, irrespective of the fact that the direct AOI signal strength is drastically different in these two cases. This opens up the possibility of quantitative measurement of the optical properties of inclusions at depth in biological tissue with no a priori knowledge of the optical properties of the surrounding media.

\section{CONCLUSIONS}

The ratio of acousto-optic signals generated in highly scattering tissue was correlated with the reduced scattering coefficient of the samples. A GaAs photorefractive crystal based interferometer, operating at a wavelength of $1064 \mathrm{~nm}$, was used to detect the acousto-optic signals generated at ultrasound focal pressures of $1.5 \mathrm{MPa}$ and $0.3 \mathrm{MPa}$ as the ultrasound pulse traversed the focus. The ratio of the magnitude of the two signals was found to increase with the reduced scattering, and this increase was related to a decrease in the phase shift induced on the light by the ultrasound as scattering is increased. In general, direct acousto-optic imaging allows for qualitative measurement of optical properties and image contrast based on optical property differences. Quantitative determination of the optical properties, however, is difficult. The signal strength is dependent on the amount of light in the AO interaction region and the optical properties of the media between this region and the detection system. In the case of photorefractive crystal based detection, the AO signal is also dependent upon the characteristics of the PRC such as the two-wave-mixing gain and optical absorption 
coefficient. Pressure contrast imaging, using the ratio of AO signals measured at two pressures, is insensitive to these parameters and is dictated primarily by the characteristics of the ultrasound pulses used and the optical properties of the acousto-optic interaction region. Detection and characterization a buried inclusion was accomplished by translating the position of the focus with respect to the sample. Measurement of the pressure ratio at the center of the inclusion allowed for the reduced scattering coefficient to be determined. Pressure contrast imaging potentially offers a relatively simple means for the quantitative assessment of tissue properties.

\section{ACKNOWLEDGEMENT}

This work was supported by the Bernard M. Gordon Center for Subsurface Sensing and Imaging Systems (CENSSIS) under the Engineering Research Centers Program of the National Science Foundation (Award number EEC-9986821).

\section{REFERENCES}

[1] V. Tuchin, Tissue Optics: Light Scattering Methods and Instruments for Medical Diagnosis (SPIE Press, Bellingham, Washington USA, 2007).

[2] D. Dolfi, and F. Micheron, "Imaging process and system for transillumination with photon frequency marking," in International Patent WO 89/00278(1989).

[3] F. A. Marks, H. W. Tomlinson, and G. W. Brooksby, "A comprehensive approach to breast cancer detection using light: photon localization by ultrasound modulation and tissue characterization by spectral discrimination," SPIE $\mathbf{1 8 8 8}$, 500-510 (1993).

[4] L. V. Wang, S. L. Jacques, and X. Zhao, "Continuous-wave ultrasonic modulation of scattered laser light to image objects in turbid media," Opt. Lett. 20, 629-631 (1995).

[5] W. Leutz, and G. Maret, "Ultrasonic modulation of multiply scattered light," Phyisca B 204, 14-19 (1995).

[6] L. V. Wang, "Mechanisms of ultrasound modulation of multiply scattered coherent light: An anylatic model," Phys. Rev. Lett. 87, 043903 (2001).

[7] A. Lev, Z. Kotler, and B. G. Sfez, "Ultrasound tagged light imaging in turbid media in a reflectance geometry," Opt. Lett. 25, 378-380 (2000).

[8] G. Yao, and L. V. Wang, "Theoretical and experimental studies of ultrasound-modulated optical tomography in biological tissue," Appl. Opt. 39, 659-664 (2000).

[9] M. Atlan, B. C. Forget, and A. C. Boccara, "Pulsed acousto-optic imaging in dynamic scattering media with heterodyne parallel speckle detection," Opt. Lett. 30, 1360-1362 (2005).

[10] T. W. Murray, L. Sui, G. Maguluri, R. A. Roy, A. Nieva, F. J. Blonigen, and C. A. Dimarzio, "Detection of ultrasound-modulated photons in diffuse media using the photorefractive effect," Opt. Lett. 29, 2509-2511 (2004).

[11] F. Ramaz, B. C. Forget, M. Atlan, and A. C. Boccara, "Photorefractive detection of tagged photons in ultrasound modulated optical tomography of thick biological tissues," Opt. Express 12, 5469-5474 (2004).

[12] X. Xu, H. Zhang, P. Hemmer, D.-k. Qing, C. Kim, and L. V. Wang, "Photorefractive detection of tissue optical and mechanical properties by ultrasound modulated optical tomography," Opt. Lett. 32, 656-658 (2007).

[13] G. Rousseau, A. Blouin, and J.-P. Monchalin, "Ultrasound-modulated optical imaging using a powerful long pulse laser," Opt. Express 16, 12577-12590 (2008). 
[14] S. Sakadzic, and L. V. Wang, "High resolution ultrasound-modulated optical tomography in biologica tissues," Opt. Lett. 29, 2770-2772 (2004).

[15] Y. Li, P. Hemmer, C. Kim, H. Zhang, and L. V. Wang, "Detection of ultrasound-modulated diffuse photons using spectral-hole burning," Opt. Express 16, 13 (2008).

[16] C. Kim, and L. V. Wang, "Multi-optical-wavelength ultrasound-modulated optical tomography: a phantom study," Opt. Lett. 32, 2285-2287 (2007).

[17] "American National Standard for the Safe Use of Laser in Health Care Facilities, ANSI Z1361.1-2000," (American National Standards Institute, New York, 2000).

[18] F. J. Blonigen, A. Nieva, C. A. Dimarzio, S. Manneville, L. Sui, G. Maguluri, T. W. Murray, and R. A. Roy, "Computations of the acoustically induced phase shifts of optical paths in acoustophotonic imaging with photorefractive-based detection," Appl. Opt. 44, 3735-3746 (2005).

[19] M. Gross, F. Ramaz, B. C. Forget, M. Atlan, A. C. Boccara, P. Delaye, and G. Roosen, "Theoretical description of the photorefractive detection of the ultrasound modulated photons in scattering media," Opt. Express 13, 7097-7112 (2005).

[20] S. Prahl, (2007), http://omlc.ogi.edu/calc/mie_calc.html.

[21] S.-R. Kothapalli, S. Sakadzic, C. Kim, and L. V. Wang, "Imaging optically scattering objects with ultrasound-modulated optical tomography," Opt. Lett. 32, 2351-2353 (2007).

[22] S.-R. Kothapalli, and L. V. Wang, "Ultrasound-modulated optical microscopy," J. of Biomed. Opt. 13, 8 (2008). 